Joseph C. Dort, M.D., F.R.C.S.C., and Ugo Fisch, M.D.

\title{
FACIAL NERVE SCHWANNOMAS
}

\begin{abstract}
A study of 26 patients with facial nerve schwannomas treated at the Unviersity Hospital of Zurich was done. The general clinical features are described, but particular emphasis is placed on tumor histologic findings, recovery of facial function after grafting, and the nature of intracranial facial nerve schwannomas. Presenting symptoms are stratified by tumor location, with facial dysfunction being commonest with intratemporal tumors, neurootologic symptoms being associated with intracranial tumors, and parotid masses being a feature of extratemporal tumors. We found no differences in tumor histology regardless of site of origin. Clinical, histologic, and radiologic evidence is reviewed, and from this evidence we conclude that intracranial facial nerve schwannomas may be particularly invasive acoustic schwannomas. Recovery of facial movement after grafting the facial nerve is not influenced by graft length or graft type. Prolonged preoperative facial dysfunction has a negative influence on recovery after grafting.
\end{abstract}

Since the first case report in 1930, many authors have written about schwannomas of the facial nerve, usually reporting single or at most a few cases. However, several larger series' have been reported ${ }^{1-6}$ that have answered many questions about presenting symptoms, diagnosis, and treatment of facial nerve schwannomas. Pulec in 1969 and $1972^{3,4}$ reviewed the literature on facial nerve schwannoma and reported his own series of cases. This author stressed the clinical features and the varied clinical presentations of facial nerve schwannomas. Based on his experience with these tumors, Pulec advocated an aggressive surgical approach in selected patients with facial nerve palsies. Conley and Janecka ${ }^{2}$ subsequently reported a series of patients with predominantly extratemporal facial nerve schwannomas and pointed out the difficulty in making a correct preoperative diagnosis. Nerve reconstruction by either free grafting or hypoglossal-facial anastomosis was done and the authors reported that the latter procedure was more reliable in their hands. Finally, the otologic medical group 1,5 have reported the largest single series' of patients with facial nerve schwannoma. The reports from this institution are unique because of the high proportion of patients with intracranial tumors.

These excellent studies notwithstanding, several questions about facial nerve schwannoma remain unanswered. First, none of the studies has reported detailed data on the return of facial function postoperatively and in particular whether nerve graft length has any influence on functional results. Second, no study has compared the histologic features of intracranial and intratemporal tumors for similarities or differences. Third, we need to know how the entity of intracranial facial nerve schwannoma fits into the spectrum of facial nerve schwannomas as a whole.

The purpose of this communication is to provide answers to these questions based on the analysis of a population of patients with facial nerve schwannoma treated at the University Hospital of Zurich between 1971 and 1988.

\section{MATERIAL AND METHODS}

The clinical records of all 26 patients with facial nerve schwannoma treated between 1971 and 1988 were collected and analyzed retrospectively. There were 16 females and 10 males with an average age of 36 years (range, 15 to 64). No patients were excluded from the study and follow-up information was available for all of the patients. Facial function was graded using the detailed evaluation of facial symmetry (DEFS) system. ${ }^{7,8}$ The average return of facial function was calculated by summing the scores for subjective global evaluation (SGE), objective global evaluation (OGE), and DEFS. This number was

Department of Otolaryngology, University Hospital, Zurich, Switzerland Reprint requests: Dr. Dort, Department of Otolaryngology, University of Manitoba, 61 Emily Street, Winnipeg, Manitoba, Canada, R3E 1Y9 Copyright $@ 1991$ by Thieme Medical Publishers, Inc., 381 Park Avenue South, New York, NY 10016. All rights reserved. 
then divided by three to yield the average return of facial function (ARF). Postoperative facial function evaluation was available for 22 of the 26 patients.

The histologic sections were also reviewed with a pathologist in order to determine if there were any histologic differences between schwannomas originating at different points along the facial nerve. We have used the conventional method of classifying facial nerve schwannomas as intracranial, intratemporal, or extratemporal. An intratemporal tumor is one in which the bulk of the lesion is confined to the temporal bone. An extratemporal tumor is a lesion that arises outside of the temporal bone in the parotid region. An intracranial facial nerve schwannoma is a tumor that arises within the internal auditory canal or cerebellopontine angle. The distribution and classification of facial nerve schwannomas in this series are shown in Table I.

\section{RESULTS}

\section{Clinical Presentation and Investigation}

Presenting symptoms and signs may be stratified into three groups: facial palsy, otologic symptoms, and parotid masses. The three types of facial nerve schwannoma are fundamentally different in their mode of presentation. Patients with intratemporal tumors presented with facial palsy $81 \%$ ( 13 of 16$)$ of the time. Five patients in this group also had conductive hearing losses. All five patients with extratemporal tumors presented with parotid masses alone. Finally, patients with intracranial schwannomas presented with sensorineural hearing losses ( 5 of 5 ), tinnitus ( 4 of 5 ), and vestibular dysfunction ( 3 of 5). Only one patient in the extratemporal group had a very mild facial weakness (DEFS, 91\%), and no patient with an intracranial schwannoma had facial weakness.

Symptoms were present for a disturbingly long time in all patient groups. Facial dysfunction was present for an average of 23 months (range, 12 to 48 ) before referral and auditory symptoms were a problem for an average of 25 months (range, 8 to 60 ). Perhaps even more surprisingly, parotid masses were present for an average of 42 months (range, 3 to 96) prior to referral for definitive treatment.

Table 1. Tumor Classification

\begin{tabular}{lrr}
\hline & No. \\
\hline Intratemporal & 16 & 8 \\
Intratemporal alone & & 4 \\
Intratemporal with extratemporal extension & & 3 \\
Intratemporal with extension into internal & & \\
$\quad$ auditory canal & & 1 \\
Intratemporal with intracranial extension & 5 & \\
Extratemporal & 5 & \\
Intracranial &
\end{tabular}

Our preoperative diagnostic workup included electroneurography (ENOG) or electromyography (EMG), or both, as well as audiometry and vestibular tests. Topognostic testing is not routinely used in our clinic, since we do not believe it is helpful in diagnosis.

Radiologic investigations were done in all patients. Polytomography and plain $\mathrm{x}$-rays were frequently used in the 1970s; however, recently high-resolution computed tomography (CT) scanning and magnetic resonance imaging (MRI) are our investigations of choice. High-resolution CT successfully mapped the facial nerve schwannoma on five of six occasions, and MRI was accurate both times it was used. More experience needs to be obtained with MRI, but it clearly has a role in the diagnosis of facial nerve schwannoma.

\section{Treatment and Operative Findings}

All 26 tumors were completely excised and the patients have been free of recurrence. The largest tumor (extratemporal) was $5 \mathrm{~cm}$ in diameter and the smallest (intracranial) was $1 \mathrm{~cm}$ in diameter. Extratemporal lesions were removed via an extratemporal (parotidectomy) approach and the intracranial tumors were removed via the transotic approach. ${ }^{9}$ Intratemporal schwannomas were removed using transmastoid, transtemporal, and combined approaches as dictated by the tumor size and location.

The schwannomas were well circumscribed grossly but several instances of microscopic transgression of resection margins were discovered by frozen section analysis, thereby necessitating additional nerve resection. None of the intracranial schwannomas involved the geniculate portion of the facial nerve. This is in contradistinction to the intratemporal tumors in which the geniculum was involved in 14 of 16 cases.

The intracranial tumors were very difficult to differentiate at the time of surgery from the more common acoustic schwannoma. In all cases the facial nerve was completely replaced with tumor and therefore the lesions were considered to be facial nerve schwannomas. Nonetheless, the eighth nerve was also usually involved and had to be resected in all of the intracranial cases.

The facial nerve had to be sacrificed in all cases except one ( $n=25$ grafts) in which the tumor was successfully dissected off the pes anserinus. Nerve grafts (greater auricular or sural nerve) were always used to reconstruct the facial nerve regardless of duration of preoperative facial palsy, patient age, or size of nerve defect. Partial nerve resection and inlay grafting was not done.

We were very interested in recovery of facial movement after nerve grafting. Graft length ranged from 1 to 10 $\mathrm{cm}$ (mean, 4.3) and both single and cable grafts were used. Table 2 shows the mean results of grafting broken down by length of graft. There was no statistical difference (Student $t$ test) between the three groups, and in fact there was a slight tendency for longer grafts to yield better 
Table 2. Mean Recovery Scores After Facial Nerve Grafting

\begin{tabular}{|c|c|c|}
\hline Graft Length ( $n=21$ grafts)* & $\begin{array}{c}\text { DEFS' } \\
(\%)\end{array}$ & $\begin{array}{c}A R F^{t} \\
(\%)\end{array}$ \\
\hline $\begin{array}{l}<2 \mathrm{~cm}(\mathrm{n}=3) \\
2 \text { to } 4 \mathrm{~cm}(\mathrm{n}=9) \\
>4 \mathrm{~cm}(\mathrm{n}=9)\end{array}$ & $\begin{array}{l}54 \pm 15 \\
51 \pm 19 \\
60 \pm 7\end{array}$ & $\begin{array}{l}52 \pm 7 \\
50 \pm 20 \\
61 \pm 10\end{array}$ \\
\hline $\begin{array}{l}\text { Cable graft }(n=6) \\
\text { Single graft }(n=15) \\
\text { Preoperative facial dysfunction } \\
\quad(n=12)\end{array}$ & $\begin{array}{l}58 \pm 5 \\
54 \pm 17 \\
58 \pm 8\end{array}$ & $\begin{array}{l}59 \pm 3 \\
54 \pm 18 \\
56 \pm 9\end{array}$ \\
\hline $\begin{array}{l}\text { No preoperative facial dysfunction } \\
(\mathrm{n}=9)\end{array}$ & $52 \pm 19$ & $54 \pm 21$ \\
\hline
\end{tabular}

*Recovery data in four patients were not available.

tARF: average recovery of function; DEFS: detailed evaluation of facial symmetry.

recovery scores. There was also no difference in outcome between cable grafts and single grafts (Table 2). The average recovery scores ranged from a low of $6 \%$ in a patient with an intracranial tumor and a $3 \mathrm{~cm}$ graft, to a high of $72 \%$ in a patient with an extratemporal tumor and an $8 \mathrm{~cm}$ graft. These recovery scores correspond to House grades III to V.

The duration of preoperative facial weakness adversely affected recovery after grafting, but this was not statistically significant. There was also no significant difference in recovery scores or recovery time between patients with and without preoperative facial dysfunction (Table 2).

\section{Complications}

The postoperative complications are shown in Table 3. One of the cerebrospinal fluid (CSF) leaks in the intratemporal group had to be sealed surgically, whereas the other two resolved spontaneously with conservative treatment. The patient with late onset of vertigo has had persistent problems and the cause of her dysfunction is unknown. Vestibular function is normal. The ulnar nerve

Table 3. Complications

\begin{tabular}{|c|c|c|}
\hline Tumor Site & Complication & Outcome \\
\hline \multirow[t]{2}{*}{ Intratemporal } & $\begin{array}{l}\text { Early CSF* leak } \\
\text { Early CSF leak }\end{array}$ & $\begin{array}{l}\text { Wound revision } \\
\text { Spontaneous } \\
\text { resolution }\end{array}$ \\
\hline & $\begin{array}{l}\text { Late onset tinnitus } \\
\text { or vertigo }\end{array}$ & $\begin{array}{l}\text { Persistent, cause } \\
\text { unknown }\end{array}$ \\
\hline \multirow[t]{3}{*}{ Intracranial } & $\begin{array}{l}\text { Early postoperative } \\
\text { headache }\end{array}$ & $\begin{array}{l}\text { Cause unknown, } \\
\text { resolved with } \\
\text { analgesics }\end{array}$ \\
\hline & Early CSF leak & $\begin{array}{l}\text { Spontaneous reso- } \\
\text { lution }\end{array}$ \\
\hline & Ulnar nerve palsy & Resolved \\
\hline
\end{tabular}

${ }^{*}$ CSF: cerebrospinal fluid. palsy was a transient problem and was due to malpositioning the patient prior to surgery.

\section{Pathologic Review}

The pathology slides from the tumors were reviewed and no differences could be seen in the histologic appearance of the schwannomas from one group to the next.

\section{DISCUSSION}

\section{Pathology}

Schwannomas are benign tumors of peripheral nerves (including cranial and spinal nerves) that originate from schwann cells. ${ }^{10-12}$ They arise from sensory nerves 12 and in clinical practice they are commonly referred to as neuromas. Malignant schwannomas occur very rarely. 13 Histologic examination of the tumors in this study revealed no differences between facial nerve schwannomas regardless of site of origin. Unlike acoustic schwannomas, which can have an unpredictable growth rate, facial nerve schwannomas appear to be uniformly slowly growing tumors. In the present series the largest tumor was $5 \mathrm{~cm}$ in diameter and some of the lesions had been present for many years before treatment.

\section{Tumor Distribution}

When discussing the experience of a single surgeon or single center with a problem like facial nerve schwannoma, it must be recognized that bias due to referral pattern and practice profile is inevitable. For example, the reports from the Otologic Medical Group ${ }^{1,5}$ are skewed toward patients with intracranial facial nerve schwannomas. In contrast, the experience of Conley and Janecka ${ }^{2}$ favors extratemporal tumors. Pulec ${ }^{3,4}$ was one of the earliest surgeons to report a larger series of patients with facial nerve schwannoma and his experience was primarily with intratemporal tumors. Our own series is relatively well balanced. The senior author (U.F.) treats a full range of facial nerve problems from the cerebellopontine angle to the parotid gland and therefore no patient selection has occurred. This is an important consideration if one is trying to observe the full spectrum of a given disease.

In our experience the majority of patients have intratemporal tumors (16 of 26), with the rest being evenly divided between intracranial and extratemporal disease. We do not consider tumors that arise in the perigeniculate area with medial extension to the internal auditory canal and cerebellopontine angle to be intracranial lesions. This tumor distribution agrees with that reported by Lipkin et $a^{6}{ }^{6}$ in their 1987 review of the world literature. 


\section{Clinical Features}

Presenting symptoms are of three major types: (1) facial palsy or paresis; (2) otologic symptoms, and (3) parotid masses. These symptoms are stratified according to tumor location, as shown in the results of this study and as reported by others. ${ }^{2,6}$ There is no "classic" presentation for these tumors as a group, 14 but each type of facial nerve schwannoma has its own characteristic signs and symptoms.

Facial weakness, when present, may be partial or complete and may be of sudden or gradual onset. We find a high correlation between facial dysfunction and intratemporal tumor location. This association is easily explained by tumor compression of the facial nerve in the unyielding fallopian canal. Otologic problems such as neurosensory hearing loss, tinnitus, and vertigo were confined to the intracranial tumor patients. None of our intratemporal tumor patients had neurosensory hearing loss attributable to the tumor.

The nonspecific presentation of intracranial facial nerve schwannoma certainly makes preoperative diagnosis difficult.2,14,15 All of our patients with intracranial facial nerve schwannomas had a preoperative diagnosis of acoustic schwannoma and the correct diagnosis was only made at the time of surgery.

\section{Diagnosis}

Accurate preoperative diagnosis of facial nerve schwannoma is important in order to advise the patient properly and plan the surgical approach. Unfortunately, it is also one of the most difficult aspects of managing patients with facial nerve schwannoma. Particularly challenging is the differentiation of intracranial facial nerve schwannomas from acoustic schwannomas. Many diagnostic tests have been recommended $3,4,15,16$ and all of our patients receive a battery of audiometric, electrophysiologic (ENOG and EMG), and neuro-otologic investigations when indicated. We, like others, have come to rely on $\mathrm{x}$-rays to diagnose tumors of the facial nerve.

Jackson et al ${ }^{17}$ suggested directing the focus of radiologic investigations to the perigeniculate region because this is the site of origin of most intratemporal facial nerve schwannomas. More recently, Inoue et $\mathrm{al}^{18}$ have addressed the problem of differentiating facial nerve schwannomas from acoustic and trigeminal schwannomas. These authors compared the CT findings of five patients with facial nerve scwhannomas to those of ten patients with acoustic schwannomas and five with trigeminal schwannomas. Unfortunately, all of the patients with facial nerve schwannomas had tumor involving the geniculate region, thereby making the diagnosis of facial nerve schwannoma relatively simple.
Intracranial facial nerve schwannomas present the major diagnostic challenge for the neuroradiologist. Latack et al ${ }^{19}$ note that the CT findings associated with a purely proximal facial nerve schwannoma are identical to those of an acoustic schwannoma.

Other diagnostic methods have been tried. Brackmann et $\mathrm{al}^{20}$ used a combination of brainstem audiometry and ENOG to help differentiate facial nerve schwannomas from acoustic schwannomas. This was a preliminary study, but the technique seemed logical and potentially useful. We have not used this method and a recent publication$^{1}$ from the Otologic Medical Group makes no reference to their current utilization of this technique. Ultrastructural analysis of fine needle aspiration biopsies has also been used to diagnose facial nerve schwannoma, ${ }^{21}$ but this technique would have little if any application for cerebellopontine angle lesions.

Our experience with correctly diagnosing intracranial facial nerve schwannomas preoperatively has been poor and has led us to question whether this entity exists as an isolated posterior fossa lesion. Our surgical findings of complete replacement of the facial nerve by tumor suggest that intracranial facial nerve schwannoma may exist, but it is also possible that these were particularly invasive acoustic schwannomas. Neely and Neblett ${ }^{22}$ found that nonacoustic cerebellopontine angle tumors were more likely to disturb facial function than invasive acoustic schwannomas. Therefore normal facial function and a posterior fossa tumor is unlikely to be due to a facial nerve schwannoma.

Friede 23 has stated that intradural facial nerve schwannomas are extremely rare and histologic studies 24 have shown that the geniculate region is predisposed to the development of facial nerve neoplasms. Radiologic studies of facial nerve schwannomas $18,19,25$ rarely show an intracranial facial nerve schwannoma without geniculate involvement. Finally, the often cited study of 600 temporal bones by Saito and Baxter 12 reveals that the "intracranial" facial nerve schwannomas found were all clearly associated with aberrant geniculate ganglion cells in the internal auditory canal.

A recent study by $\mathrm{O}^{\prime}$ Donoghue et $\mathrm{a}^{1}{ }^{1}$ reports on a large series of patients, many of whom had posterior fossa disease. These patients presented in a manner identical to acoustic schwannoma patients and in fact $36 \%$ were so diagnosed preoperatively. Unfortunately, the authors do not comment on what criteria were used subsequently to make the diagnosis of facial nerve schwannoma. O'Donoghue et al conclude that it is clear that most segments of the facial nerve are affected equally. To us, it is far from clear based on our surgical observations, histologic evidence, 12,23,24 radiologic studies, $18,19,25$ and the clinical reports of others, 2,6,22,27 all of which suggest that pure intracranial facial nerve schwannomas are very uncommon tumors. Given the very different clinical behavior of 
intracranial facial nerve schwannomas, perhaps they are a separate entity with a different histogenesis from the more common intratemporal and extratemporal lesions.

\section{Facial Rehabilitation}

Surgery is the only treatment for facial nerve schwannoma and therefore patients are left with permanent facial dysfunction after treatment because tumor removal almost always requires total nerve resection. Partial nerve resection and the use of inlay grafts have given consistently poor results in our hands; therefore we prefer to resect the nerve and interpose a graft. O'Donoghue et al ${ }^{1}$ were able to preserve at least $50 \%$ of the facial nerve in $25 \%$ of their patients, but this has not been the experience of others. $3,4,16,26,27$

The best means of reconstructing the facial nerve has been a controversial subject. Spector ${ }^{28}$ has stated a preference for short cable grafts over long cable grafts for reconstruction of the facial nerve. Spector also prefers to use a hypoglossal-facial anastomosis instead of long nerve grafts and he also condemns the use of sutureless anastomoses and fibrin glue in nerve repairs. We believe that a nerve interposition graft gives the best return of facial function and that the length of the graft has no influence on the eventual return of function. The recovery profiles after grafting in this study show good return of function regardless of graft length or whether cable or single grafts were used. We did notice a trend to poorer recovery in patients with long-standing palsy, a finding noted by others. 16 Hypoglossal-facial anastomosis also yields good results, particularly in patients with long-standing preoperative facial palsy, 16,29 but we have not needed to use it in our facial nerve schwannoma patients. We commonly make use of sutureless techniques and fibrin glue, especially for an intratemporal nerve anastomosis, and have found no adverse effects on nerve regeneration. In addition, we also protect the anastomotic site with a small sheet of Cargile membrane.

\section{SUMMARY}

Facial nerve schwannomas are unusual tumors that arise primarily in the geniculate region from sensory fibers of the facial nerve. Intratemporal facial nerve schwannomas behave differently from intracranial and extratemporal tumors. Surgical experience and review of the radiologic and histologic evidence have led us to conclude that isolated intracranial facial nerve schwannoma is very rare and may represent an invasive acoustic schwannoma. We also conclude that interposition grafting is the best reconstructive technique when there is a viable proximal and distal facial nerve stump and that graft length has no influence on eventual recovery.
The problem of accurate preoperative differentiation of intracranial facial nerve schwannoma from acoustic schwannoma remains. It is those patients with normal facial movements in the presence of an intracranial facial nerve schwannoma who may be better served by having radiologic follow-up and deferral of surgery until facial dysfunction or other problems supervene.

\section{REFERENCES}

1. O'Donoghue GM, Brackmann DE, House JW, Jackler RK: Neuromas of the facial nerve. Am J Otol 10:49-54, 1989

2. Conley J, Janecka I: Schwann cell tumors of the facial nerve. Laryngoscope 84:958-962, 1974

3. Pulec J: Facial nerve tumors. Ann Otol Rhinol Laryngol 78:962982, 1969

4. Pulec J: Symposium on ear surgery. II. Facial nerve neuroma. Laryngoscope 82:1160-1176, 1972

5. Nelson RA, House WF: Facial nerve neuroma in the posterior fossa: surgical considerations. In Graham MD, House WF (eds): Disorders of the Facial Nerve. New York: Raven Press, 1982, pp. 403-406

6. Lipkin A, Coker N, Jenkins H, Alford B: Intracranial and intratemporal facial neuroma. Otolaryngol Head Neck Surg 96:71-79, 1987

7. Fisch U: Surgery for Bells palsy. Arch Otolaryngol 107:1-11, 1981

8. Burres S, Fisch U: The comparison of facial grading systems. Arch Otolaryngol Head Neck Surg 112:755-758, 1986

9. Fisch U, Mattox D: Microsurgery of the skull base. Stuttgart: Georg Thieme Verlag, 1988, pp 74-119

10. Schuknecht H F: Pathology of the Ear. Cambridge: Harvard University Press, 1974, pp 425-441

11. Batsakis JG: Pathology of Head and Neck Tumours. 1974, pp 231-249

12. Saito H, Baxter A: Undiagnosed intratemporal facial nerve neurilemomas. Arch Otolaryngol 95:415-419, 1972

13. Ul Hasan N, Kazi T: Malignant schwannoma of facial nerve. $J$ Pediatr Surg 21:926-928, 1986

14. Neely JG, Alford B: Facial nerve neuromas. Arch Otolaryngol 100:298-301, 1974

15. Pillsbury H, Price H, Gardiner L: Primary tumors of the facial nerve: Diagnosis and management. Laryngoscope 93:10451048,1983

16. May M: The Facial Nerve. New York: Thieme, 1986

17. Jackson CG, Glasscock M, Hughes G, Sismanis A: Facial paralysis of neoplastic origin: Diaagnosis and management. Laryngoscope 90:1581-1595, 1980

18. Inoue $\mathrm{Y}$, Tabuchi $\mathrm{T}$, Hakuba A, et al; Facial nerve neuromas: CT findings. J Comput Assit Tomogr 11:942-947, 1987

19. Latack JT, Gabrielsen TO, Knake JE, et al: Facial nerve neuromas: Radiologic evaluation. Radiology 149:731-739, 1983

20. Brackmann DE, House JW, Selters W: Auditory brainstem responses in facial nerve neurinoma diagnosis. In Graham M, House W (eds.): Disorders of the Facial Nerve. New York: Raven Press, 1982, pp 87-89

21. Navas Palacios J J, De Agustin de Agustin PP, De Isso Heros FA, et al: Ultrastructural diagnosis of facial nerve schwannoma using fine needle aspiration. Acta Cytol 27:441-445, 1983

22. Neely JG, Neblett CR: Differential facial nerve function in tumors of the internal auditory meatus. Ann Otol Rhinol Laryngol 92: 39-41, 1983

23. Friede RL: Pathology of intracranial tumors producing facial palsy. In Fisch U (ed): Facial Nerve Surgery. Birmingham, AL: Aesculapius Publishing, 1977, pp 470-473

24. Fisch U, Ruttner J: Pathology of intratemporal tumors involving the facial nerve. In Fisch U (ed): Facial Nerve Surgery: Proceedings of the Third International Symposium. Birmingham, AL: Aesculapius Publishing, 1977, pp 448-456 
25. Valvanis A, Schubiger O, Naidich TP: Neurinomas of the facial nerve. In Clinical Imaging of the Cerebello-Pontine Angle. Springer-Verlag; 1987, pp 126-130

26. Janecka I, Conley J: Primary neoplasms of the facial nerve. Plast Reconstr Surg 79:177-183, 1987

27. Horn K, Crumley R, Schindler R: Facial neurlemmomas. Laryngoscope 91:1326-1331, 1981

28. Spector J G: Mimetic surgery for the paralyzed face. Laryngoscope 95:1494-1522, 1985
29. Conley J, Baker DC: Hypoglossal-facial anastomosis for reinnervation of the paralyzed face. Plast Reconstr Surg 63:63-72, 1979

We would like to thank the Manitoba Medical College Fund and the Manitoba Faculty Fund for providing financial assistance to Dr. Dort. We also thank Prof. M. Makec, Department of Pathology, University Hospital, Zurich, for his help in reviewing the tumor histology. 